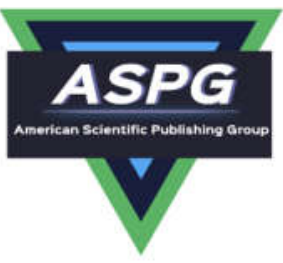

\title{
The Concept of Neutrosophic Limits in Real Sequences
}

\author{
Huda E. Khalid ${ }^{1 *}$, Ahmed K. Essa ${ }^{2}$ \\ 1,2 Telafer University, Scientific Affairs and Cultural Relations Department, Telafer, Iraq; hodaesmail@yahoo.com \\ * Correspondence: hodaesmail@yahoo.com
}

\begin{abstract}
The theory of neutrosophic limits is the natural step before developing the theory of neutrosophic continuous functions and developing the theory of neutrosophic differentiation. The goal of this work is to construct a new definition of the neutrosophic limits for real sequences using the distance as a linear operator. Some new theorems are presented to cover the theoretical notions of this topic and an illustrative example is presented to help the reader understanding the notions of this article.
\end{abstract}

Keywords: Neutrosophic Limits, Neutrosophic Convergence, Real Sequences.

\section{Introduction}

The essential concepts that should be extended in neutrosophic theory are the fuzzy limits, fuzzy continuity, and fuzzy derivatives. Neutrosophic researchers must extend the usual concept of the conventional limit of a sequence. Furthermore, the main differences between neutrosophic theory and fuzzy theory are that any notion in neutrosophic theory say $\langle A\rangle$ together with its counteractive $\langle$ anti $A\rangle$ and with their spectrum of neutralities $\langle$ neut $A\rangle$ in between them (i.e. ideas supporting neither $\langle A\rangle$ nor $\langle$ antiA $>$ ). The $\langle$ neut $A\rangle$ and $\langle$ antiA $>$ ideas together are referred to as $\langle\operatorname{non} A\rangle$. Neutrosophic logic is generalization of the fuzzy logic. In neutrosophic logic a proposition has a degree of truth (T), a degree of indeterminacy (I), and a degree of falsity (F), where T, I, F are standard or non-standard subsets of ${ }^{-} 0,1^{+}$[ [1]. The neutrosophic logic was established by F. Smarandache [2]. In this paper, the authors introduce pioneering work called neutrosophic limits for real-sequences using the linear operator. The first improvement in the topic about neutrosophic limits was by F. Smarandache in his first edition of neutrosophic calculus [3], the second attempt to determine the concept of functional limits, introduce the binomial factorial theorem, and specify the indeterminate values of neutrosophic calculus were by Huda E. Khalid et al. [4,14]. There are many additional works by Huda E. Khalid concerning specifying the type of indeterminacy regarded to the (over, off, under) neutrosophic theory $[5,6,7,10]$, also she worked to improve the concept of neutrosophic geometric programming $[8,9,11,12,13]$, those previous works did not cover the limits and the continuity of real sequences which regards as the basic concepts of mathematical analysis. This paper represents the first attempt in this direction.

\section{Basic concepts}

\subsection{Definition [15]}


Suppose $R=(-\infty, \infty), R^{+}=[0, \infty)$ and $R^{++}=(0, \infty)$, assume that $r \in R^{+}$and let $l=\left\{a_{i} \in \mathrm{R} ; i=1,2, \ldots\right\}$ be a sequence of real numbers.

\subsection{Definition [15]}

(a) A real number " $a$ " is called an $r$-limit of a sequence $l$ (it is denoted by $a=r-\lim _{i \rightarrow \infty} a_{i}$ or $a=r-\operatorname{liml}$ ) if for any $\varepsilon \in R^{++}$, the inequality $\left|a-a_{i}\right|<r+\varepsilon$ is valid for almost all $a_{i}$, i.e., there is such $n$ that for any $i>n$, we have $\left|a-a_{i}\right|<r+\varepsilon$.

(b) A sequence $l$ that has an $r$ - limit is called $r$ - convergent and it is denoted by $l \longrightarrow r^{a}$. Informally, $a$ is an $\mathrm{r}$ limit of a sequence $l$ for an arbitrarily small $\varepsilon$, the distance between $a$ and all but a finite number of elements from $l$ is smaller than $r+\varepsilon$. In other words, a number $a$ is an $r$ - limit of a sequence $l$ if for any $\varepsilon \in R^{++}$, almost all $a_{i}$ belong to the interval $(a-r-\varepsilon, a+r+\varepsilon)$. Hence, $r$ becomes a measure of convergence for $l$.

\subsection{Compare Between Fuzzy and Neutrosophic Logics [1,7]}

The neutrosophic connections have a better truth-value definition approach to the real-world systems than the fuzzy connections. They are defined on triple non-standard subsets included in the non-standard interval $]^{-} 0,1^{+}[$, while in fuzzy theory they are defined on the interval $[0,1]$. $n_{-}$sup is not limited to 1 , but it's aggrandized to a monad $m\left(3^{+}\right)$; similarly, $n_{-}$inf maybe as low as $m\left({ }^{-} 0\right)$, not as 0 . A paradox, which is simultaneously true and false, cannot be evaluated in fuzzy logic, because the sum of the combinations should add up to 1 , but it is allowed in neutrosophic theory because of the thought of contradictory in neutrosophic logic may be $(1,1,1)$. In dissenting to the fuzzy theory, if an assumption $<\mathrm{A}>$ is $\mathrm{t} \%$ true, doesn't necessarily mean it is $(100-t) \%$ false. A better tactic is $t \%$ true, $i \%$ indeterminate, and $f \%$ false, as in intuitionistic fuzzy logic, whereas $t \in T, i \in I, f \in F$. More general, with $n_{-}$sup $\leq 3^{+}$and $n_{-}$inf $\geq{ }^{-} 0$.

\section{Neutrosophic Limits of Sequences}

This section presents basic steps to define the neutrosophic limits for real sequences using linear operator.

\subsection{The Behavior of limits in Neutrosophic Environment}

To put forward the concept of neutrosophic convergence, one must compare the differences between the fuzzy convergent in Definition 2.2 with the neutrosophic notion, the condition $\left|a-a_{i}\right|<r+\varepsilon$ given in Definition 2.2 is not enough to maintain the neutrosophic convergent of any real number $a$ to a sequence of real numbers $l$. In fact, we need two additional conditions related with $p\left(a, a_{i}\right)<r+\varepsilon$. Any neutrosophic convergence need three joints, the first hinge is the neutrosophically convergent which is somehow likely fuzzy convergent but this indeed could not be enough to lead to neutrosophic convergence, the second hinge is anti (a) which must exist and satisfy $\mid$ anti $(a)-a_{i} \mid>r+\varepsilon$, and neut(a) will be the final hinge. Logically with respect to the neutrosophic notion, anti (a) should satisfy the divergent from the sequence $l$, while neut $(a)$ is any real number that is neither convergent to $l$ nor divergent, in other words, neut $(a)$ is situated in the location between $a$ \& anti $(a)$, so the distance between neut (a) and any element $a_{i} \in l$ could not be less than $r+\varepsilon$ and could not be greater than $r+\varepsilon$. Consequently, the distance between neut $(a)$ and any element of $l$ must be equal to $r+\varepsilon$ and this only happens when neut $(a)$ becomes as sequence of neutralities say $c=\left\{c_{1}, c_{2}, c_{3}, \ldots\right\}$, where $c_{i}$ is neut $(a)$ corresponding to the value of $a_{i} \in l$. The above inspired us the following definition

\subsection{Definition}


Let $a$ be a real number, $b$ a real number or an interval of real numbers, and c be a set of real numbers. Then $a$ is called an $r$-neutrosophic limit of a sequence $l$, referred to as $a=r-N \lim _{i \rightarrow \infty} a_{i}$ or denoted by $a=r-N \lim l$, if for any $\varepsilon \in R^{++}$the following inequalities are satisfied together:

$1-\left|a-a_{i}\right|<r+\varepsilon$

2- $\left|b-a_{i}\right|>r+\varepsilon \quad$ where $b=\operatorname{anti}(a)$ [i.e. $b$ is the opposite or negation of $a$ ],

3- $\left|c_{i}-a_{i}\right|=r+\varepsilon \quad$ where $c=$ neut $(a)$ [i.e. here $c=\left\{c_{1}, c_{2}, c_{3}, \ldots\right\}, c_{i}$ is neut $(a)$ corresponding to the value of $a_{i}$ ] also neut $(a)$ means neither $a$ nor $b$.

Note that $p\left(a, a_{i}\right)<r+\varepsilon$ is valid for almost all $a_{i}$, (i.e. there is such $n$ that for any $i>n$, we have $p\left(a, a_{i}\right)<r+$ $\varepsilon)$. Also $p\left(b, a_{i}\right)>r+\varepsilon$ is valid for almost all elements of $a_{i}$, (i.e. there is such $m$ that for any $i>m$, we have $\left.p\left(b, a_{i}\right)>r+\varepsilon\right)$. Moreover, for the neutral of $a$ there is a set of elements $c=\left\{c_{1}, c_{2}, c_{3}, \ldots\right\}$ that satisfies the inequality $p\left(c_{i}, a_{i}\right)=r+\varepsilon$ ( i.e. $c_{1}$ represents the neut $(a)$ corresponding to the element $a_{1} \in l, c_{2}$ represent the neut (a) corresponding to the element $a_{2} \in l$ and so on). In this case, the sequence $l$ is $r$-neutrosophically convergent to $a$ and it is denoted by $l \stackrel{N}{\longrightarrow}{ }_{r} a$.

In other words, the element $a$ is $r-$ Nlimit of a sequence $l$ if for an arbitrarily small $\varepsilon \in R^{++}$, the distance between $a$ and all but a finite number of elements from $l$ is less than $r+\varepsilon$, at the same time there is another element $b$ (it could be an interval $\left.b=\left(b_{1}, b_{2}\right)\right)$ that the distance between $b$ and all but finite number of elements from $l$ is greater than $r+\varepsilon$, simultaneously for any element of $l$ say $a_{i}$ there is a corresponding element $c_{i}$ that the distance between $a_{i} \& c_{i}$ is equal to $r+\varepsilon$.

It is obvious that almost all $a_{i}$ belong to the interval $(a-r-\varepsilon, a+r+\varepsilon)$, at the same time those almost all $a_{i}$ do not belong to the interval $(b-r-\varepsilon, b+r+\varepsilon)$ while for each element $a_{i} \in l$ there is one and only one element of a sequence $c$ satisfy $a_{i}=c_{i}-r-\varepsilon$.

\section{Some Theoretical Results for Neutrosophic Limits}

This section presents some results on neutrosophic limits.

\subsection{Theorem}

Let $a, b$ be any two real numbers, and $c=\left\{c_{1}, c_{2}, c_{3}, \ldots\right\}$ be a set of real numbers, suppose that $r \in R^{+}$, and let $l=$ $\left\{a_{i} \in \mathrm{R} ; i=1,2, \ldots\right\}$ be a sequence of real numbers. If $r=0$ (i.e. $a=0-N$ limit $l$ ), then for any $\varepsilon \in R^{++}$the following inequalities hold:

$\left|a-c_{i}\right|<2 \varepsilon$

$\left|b-c_{i}\right|<2 \varepsilon$

\section{Proof}

Apply $r=0$ to the inequalities of Definition (3.2), we get

$\left|a-a_{i}\right|<\varepsilon$

$\left|b-a_{i}\right|>\varepsilon$

$\left|c_{i}-a_{i}\right|=\varepsilon$ 
From equality (3),

$c_{i}-a_{i}=\mp \varepsilon \rightarrow a_{i}=c_{i} \pm \varepsilon$

Return to the inequality $\left|a-a_{i}\right|<\varepsilon$ and by using the definition of absolute value, we will get

$0<a-a_{i}<\varepsilon \&-\varepsilon<-\left(a-a_{i}\right)<0$

Applying (4) to the above inequalities, will get the following inequalities,

$0<a-c_{i}<2 \varepsilon \&-2 \varepsilon<-\left(a-c_{i}\right)<0$,

which imply that, $\left|a-c_{i}\right|<2 \varepsilon$,

Track the same above way to prove that,

$\left|b-c_{i}\right|<2 \varepsilon$

The proof is complete.

\subsection{Theorem}

Let $a=r-N \lim l$, then $a=q-N \lim l$, at $q-r=\varepsilon_{1}$ where $\varepsilon_{1} \in R^{++}$.

\section{Proof}

Since $a=r-N \lim l$, the following inequalities are holding together:

$\left|a-a_{i}\right|<r+\varepsilon$

$\left|b-a_{i}\right|>r+\varepsilon$

$\left|c_{i}-a_{i}\right|=r+\varepsilon$

Since $q-r=\varepsilon_{1}$, it follows that $q>r$ and hence, $q+\varepsilon>r+\varepsilon$. But from inequality (5) we have,

$r+\varepsilon>\left|a-a_{i}\right| \rightarrow\left|a-a_{i}\right|<q+\varepsilon$.

Therefore, the first inequality of Definition (3.2) is holds for $q$.

From equality (7) we have, $r=\left|c_{i}-a_{i}\right|-\varepsilon$

$\Rightarrow q-\varepsilon_{1}=\left|c_{i}-a_{i}\right|-\varepsilon \Rightarrow\left|c_{i}-a_{i}\right|=q-\varepsilon_{1}+\varepsilon$,

Reset $-\varepsilon_{1}+\varepsilon$ as $\varepsilon_{2}$

$\therefore\left|c_{i}-a_{i}\right|=q+\varepsilon_{2}$, which means that the equality of Definition (3.2) is holding for q.

For the second inequality of Definition (3.2),

$\left|b-a_{i}\right|>r+\varepsilon=q-\varepsilon_{1}+\varepsilon$

reset $-\varepsilon_{1}+\varepsilon$ as $\varepsilon_{2} \Rightarrow\left|b-a_{i}\right|>q+\varepsilon_{2}$.

So, all restricted conditions of Definition (3.2) are holds for $q$. 
$\therefore a=q-N \lim l$,

\subsection{Note}

1- We can re-express Definition (3.2) in the following alternative way.

A number $a$ represents the neutrosophic limit for sequence $l$ if the triple $(a, b, c)$ satisfies

$a=r-N$ lim $l$ ( $a$ is an $r-$ Nlimit of l for some $r \in R^{+}$)

$b \neq r-$ Nlim $l \quad(b$ is not $r-$ Nlimit of $l)$

$c \nRightarrow r-N$ lim $l$ (c is niether $r-N$ limit of $l$ nor not $r-N$ limit of $l$ ).

2- A sequence $l$ is neutrosophically convergent to $a$ if $l$ has a neutrosophic limit to $a$.

\section{Numerical Example}

Let $a=1$ and $l=\left\{\frac{1}{i}\right\}$ where $i$ is the set of all-natural numbers. Test if $a$ is $2-$ Nlimit of the sequence $l$. What about the convergence of $l$ to $a=2.5$ ?

Solution:

Here $a=1, r=2$, and let $\varepsilon=0.1$, it is obvious that $p\left(1, a_{i}\right)<r+\varepsilon \Rightarrow p\left(1, a_{i}\right)<2.1$ for almost all elements of $l$. The value of $b=\operatorname{anti}(a)$ for $a=1, r=2, \varepsilon=0.1$ is $b=(3.1, \infty)$, while the sequence $c=$ neut $(a)=$ $\{3.1,2.6,2.4333, \ldots$.$\} , i.e. p\left(3.1, a_{1}\right)=2.1 \Rightarrow a_{1}=1$ which is the first element in $l, p\left(2.6, a_{2}\right)=2.1 \Rightarrow a_{2}=0.5$. which is the second element in $l$, also $p\left(2.4333, a_{3}\right)=2.1 \Rightarrow a_{3}=0.3333=\frac{1}{3}$ the third element in $l$, it is clear that the sequence $c=$ neut $(a)$ satisfies the equality $\left|c_{i}-a_{i}\right|=r+\varepsilon$, here $r+\varepsilon=2.1$. Consequently, $a=1$ is $2-$ $N$ limit of $l$ (i.e. $1=2-N \lim l$ ). But $a=2.5$ is not $2-$ Nlimit of $l$ since at $\varepsilon=0.1$ this implies that $\left|2.5-\frac{1}{i}\right|>$ 2.1 for almost all values of $i$ except $i=1$ and $i=2$, this is a contradict to the first inequality of Definition (3.2), (i.e. $2.5 \neq 2-$ Nlim $l$ ).

\section{Conclusions}

It is well known that many processes of mathematics, such as differentiation and integration, demand the use of limits. This paper presented the definition of neutrosophic limits of real sequence $l$ using the distance as a linear operator. Moreover, two theorems for the sake of the theoretical part of this paper were proved. The notions of this paper came as necessary first step to develop the notion of neutrosophic differentiation in the real space $R$ and the neutrosophic convergent.

Funding: "No external funding has been received for this research."

Conflicts of Interest: "The authors declare that there is no conflict of interest."

\section{References}


[1] F. Smarandache., Neutrosophy, "A New Branch of Philosophy, Multiple-Valued Logic / An International Journal," USA, ISSN 1023-6627, Vol. 8, No. 3, pp. 297-384, 2002.

[2] F. Smarandache., "An Introduction to Neutrosophy, Neutrosophic Logic, Neutrosophic Set, and Neutrosophic Probability and Statistics," First International Conference on Neutrosophy, Neutrosophic Logic, Set, Probability and Statistics December 1-3, 2001 the University of New Mexico, 2001.

[3] F. Smarandache, "Neutrosophic Precalculus and Neutrosophic Calculus," Second enlarged edition, EuropaNova asbl Clos du Parnasse, 3E 1000, Bruxelles Belgium, European Union, 2015.

[4] F. Smarandache \& Huda E. Khalid, "Neutrosophic Precalculus and Neutrosophic Calculus," Second enlarged edition, Pons asbl 5, Quai du Batelage, Brussels, Belgium, European Union, 2018.

[5] F. Smarandache, H. E. Khalid \& A. K. Essa, "Neutrosophic Logic: the Revolutionary Logic in Science and Philosophy," Proceedings of the National Symposium, EuropaNova, Brussels, 2018.

[6] F. Smarandache, H. E. Khalid \& A. K. Essa, "A New Order Relation on the Set of Neutrosophic Truth Values," Book chapter of New Trends in Neutrosophic Theory and Applications, Pons asbl 5, Quai du Batelage, Brussels, Belgium, European Union, 2016.

[7] F. Smarandache, H. E. Khalid, A. K. Essa, M. Ali, “The Concept of Neutrosophic Less Than or Equal To: A New Insight in Unconstrained Geometric Programming," Critical Review, Volume XII, pp. 72-80, 2016.

[8] H. E. Khalid, "An Original Notion to Find Maximal Solution in the Fuzzy Neutrosophic Relation Equations (FNRE) with Geometric Programming (GP)", Neutrosophic Sets and Systems, vol. 7, pp. 3-7, 2015.

[9] H. E. Khalid, "The Novel Attempt for Finding Minimum Solution in Fuzzy Neutrosophic Relational Geometric Programming (FNRGP) with (max, min) Composition", Neutrosophic Sets and Systems, vol. 11, pp. 107-111, 2016.

[10] H. E. Khalid, F. Smarandache, \& A. K. Essa, "The Basic Notions for (over, off, under) Neutrosophic Geometric Programming Problems," Neutrosophic Sets and Systems, 22, pp.50-62, 2018.

[11] H. E. Khalid, "Geometric Programming Dealt with a Neutrosophic Relational Equations Under the ( $\max -\min )$ Operation,"Neutrosophic Sets in Decision Analysis and Operations Research, chapter four. IGI Global Publishing House, 2020.

[12] H. E. Khalid, "Neutrosophic Geometric Programming (NGP) with (max-product) Operator, An Innovative Model,"Neutrosophic Sets and Systems, vol. 32, pp.269-281, 2020.

[13] H. E. Khalid, "Neutrosophic Geometric Programming (NGP) with (max-product) Operator, An Innovative Model", Neutrosophic Sets and Systems, vol. 32, pp.454-463, 2020.

[14] H. E. Khalid, F. Smarandache, \& A. K. Essa, "A Neutrosophic Binomial Factorial Theorem with their Refrains,"Neutrosophic Sets and Systems, 14, 50-62, 2016.

[15] M. Burgin, O. Duman," Approximations by linear operators in spaces of fuzzy continuous functions," Positivity 15,pp.57-72, 2011. DOI 10.1007/s11117-009-0041-4. 